\title{
Pathogen-Host Analysis Tool (PHAT): an Integrative Platform to Analyze Pathogen-Host Relationships in Next-Generation Sequencing Data
}

Christopher M. Gibb ${ }^{1,2 *, * *}$ (chris.gibb@outlook.com), Robert Jackson ${ }^{1,3 *}$

(rjackso1@1akeheadu.ca), Sabah Mohammed² (sabah.mohammed@lakeheadu.ca), Jinan

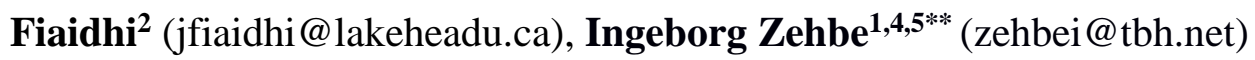

${ }^{1}$ Probe Development and Biomarker Exploration, Thunder Bay Regional Health Research

Institute, Thunder Bay, Ontario, Canada

${ }^{2}$ Department of Computer Science, Lakehead University, Thunder Bay, Ontario, Canada

${ }^{3}$ Biotechnology Program, Lakehead University, Thunder Bay, Ontario, Canada

${ }^{4}$ Department of Biology, Lakehead University, Thunder Bay, Ontario, Canada

${ }^{5}$ Northern Ontario School of Medicine, Lakehead University, Thunder Bay, Ontario, Canada

\section{*These authors contributed equally to this work}

\section{**Corresponding Authors:}

Software Developer: Christopher M. Gibb; chris.gibb@outlook.com; 1-807-684-7248

Principal Investigator: Ingeborg Zehbe, PhD, DSc; zehbei@tbh.net; 1-807-684-7246 


\section{Abstract \\ Summary}

The Pathogen-Host Analysis Tool (PHAT) is an application for processing and analyzing nextgeneration sequencing (NGS) data as it relates to relationships between pathogen and host organisms. Unlike custom scripts and tedious pipeline programming, PHAT provides an integrative platform encompassing raw and aligned sequence and reference file input, quality control (QC) reporting, alignment and variant calling, linear and circular alignment viewing, and graphical and tabular output. This novel tool aims to be user-friendly for life scientists studying diverse pathogen-host relationships.

\section{Availability and Implementation}

The project is publicly available on GitHub (https://github.com/chgibb/PHAT) and includes convenient installers, as well as portable and source versions, for both Windows and Linux (Debian and RedHat). Up-to-date documentation for PHAT, including user guides and development notes, can be found at https://chgibb.github.io/PHATDocs/. We encourage users and developers to provide feedback (error reporting, suggestions, and comments) using GitHub Issues.

\section{Contact}

Lead software developer: chris.gibb@outlook.com 


\section{Introduction}

While the ease of producing and accessing next-generation sequencing (NGS) data has grown significantly, bottlenecks still exist in its processing and analysis. Alignment algorithms, particularly for short-read alignment and the tools that implement them, have matured to the point that they no longer present the major hurdle in the data analysis process ( $\mathrm{Li}$ and Homer, 2010). The availability of fast and user-friendly tools has become the limiting factor (Milne et al., 2010). There are excellent tools which perform one or several discrete functions in the same domain, e.g., Bowtie2 (Langmead and Salzberg, 2012) and SAMtools (Li et al., 2009), however all-in-one type platforms can offer a breadth of features that help address barrier-to-entry (i.e., the ease in which users can setup and perform analyses). While integrative multi-tool platforms such as Comparative Genomics (CoGe) (Lyons and Freeling, 2008), VirBase (Li et al., 2014), Pathogen-Host Interaction Data Integration and Analysis System (PHIDIAS) (Xiang et al., 2007), Galaxy (Afgan et al., 2016), and Unipro UGENE (Okonechnikov et al., 2012) exist, all are server or cloud-based, or maintained by relatively small communities. The rather small scale of the infrastructure behind these projects and their cloud-based nature, introduce roadblocks in the transfer of data to and from their servers ( $\mathrm{Li}$ and Homer, 2010). One solution to a problem such as this is to establish an onsite computational cluster, but that can add further barrier-to-entry for data analysis due to technical and infrastructure requirements.

We sought to develop the Pathogen-Host Analysis Tool (PHAT) to alleviate these issues by presenting an easy-to-setup and easy-to-use platform for life scientists conducting pathogenhost NGS analysis on common desktop computing hardware (e.g., Windows). 


\section{Features}

Pathogen-host NGS analysis typically begins with high-throughput sequencing output files, containing experimentally relevant nucleic acid read information. PHAT provides a platform for analyzing these output data, with a focus on pathogen-host relationships (Figure 1). DNA read files are input into PHAT as FASTQ files (Cock et al., 2010), comprised of sequence reads with per base nucleotide identities and quality scores, or pre-aligned SAM/BAM files (Li et al., 2009) generated via cloud-based tools such as Galaxy (Afgan et al., 2016). Quality control analysis can be performed on individual files, with graphical and numeric quality control reports generated. Reference genomes input as FASTA files must be indexed before they can be visualized or used for analysis. Once a pair of forward and reverse reads, paired FASTQ files, and a reference genome have been input, alignment of the paired reads against the reference can occur. PHAT also supports unpaired alignment and visualization of pre-aligned sequences.

The core functions of the PHAT platform as well as FASTQ quality control, sequence alignment, and its automated analyses are performed through well-known, established implementations. Namely, quality control scoring for high-throughput sequencing data is performed by FastQC (https://www.bioinformatics.babraham.ac.uk/projects/fastqc/), sequence alignment by Bowtie2 (Langmead and Salzberg, 2012), alignment visualization by pileup.js (Vanderkam et al., 2016), circular genome visualization based on our enhancements to AngularPlasmid (http://angularplasmid.vixis.com/) which we make available as a new project called ngPlasmid (https://github.com/chgibb/ngPlasmid), and automated variant calling by VarScan 2 (Koboldt et al., 2012). The graphical user interface of the application, based on GitHub's Electron project (http://electron.atom.io/docs/) operates in a client-server based architecture. Each 
window of the application acts as a client, communicating with a background server process. The server process manages the saving of and propagation of workspace data. This server process also manages the spawning of other processes used in the application such as sequence alignment and quality control report generation. This mechanism allows other processes to act as threads, allowing the flow of data to and from the application window that invoked it and the spawned process itself. On systems with limited power, the server process can limit the number of concurrently running processes as well as the amount of data propagated between application windows to reduce memory and CPU usage. Application windows which use other processes for operations or generation of data (e.g., Bowtie2) utilize a pipeline, requesting the spawning of new processes as others close and passing data from process-to-process to arrive at the result (e.g., the conversion of data from BAM format, to a sorted, indexed BAM). The server process, as well as the application windows themselves are implemented in TypeScript. These application windows can be conveniently undocked from the main toolbar as per user requirements.

\section{Conclusion}

With the development of PHAT, we aim to bring simple-to-use, cross-platform NGS analysis to off-the-shelf hardware for life scientists studying pathogen-host relationships. In our own lab, we study human papillomavirus type 16 (HPV16) variants and their tumourigenicity in human skin using NGS (Jackson et al., 2016), but PHAT can be applied to a wide-variety of pathogen-host relationships (e.g., genotyping of microbes such as viruses, bacteria, and protozoans from host NGS samples). We plan to actively develop, update, and support PHAT, with auto-updating features already included, in anticipation of building an active user and developer community. 

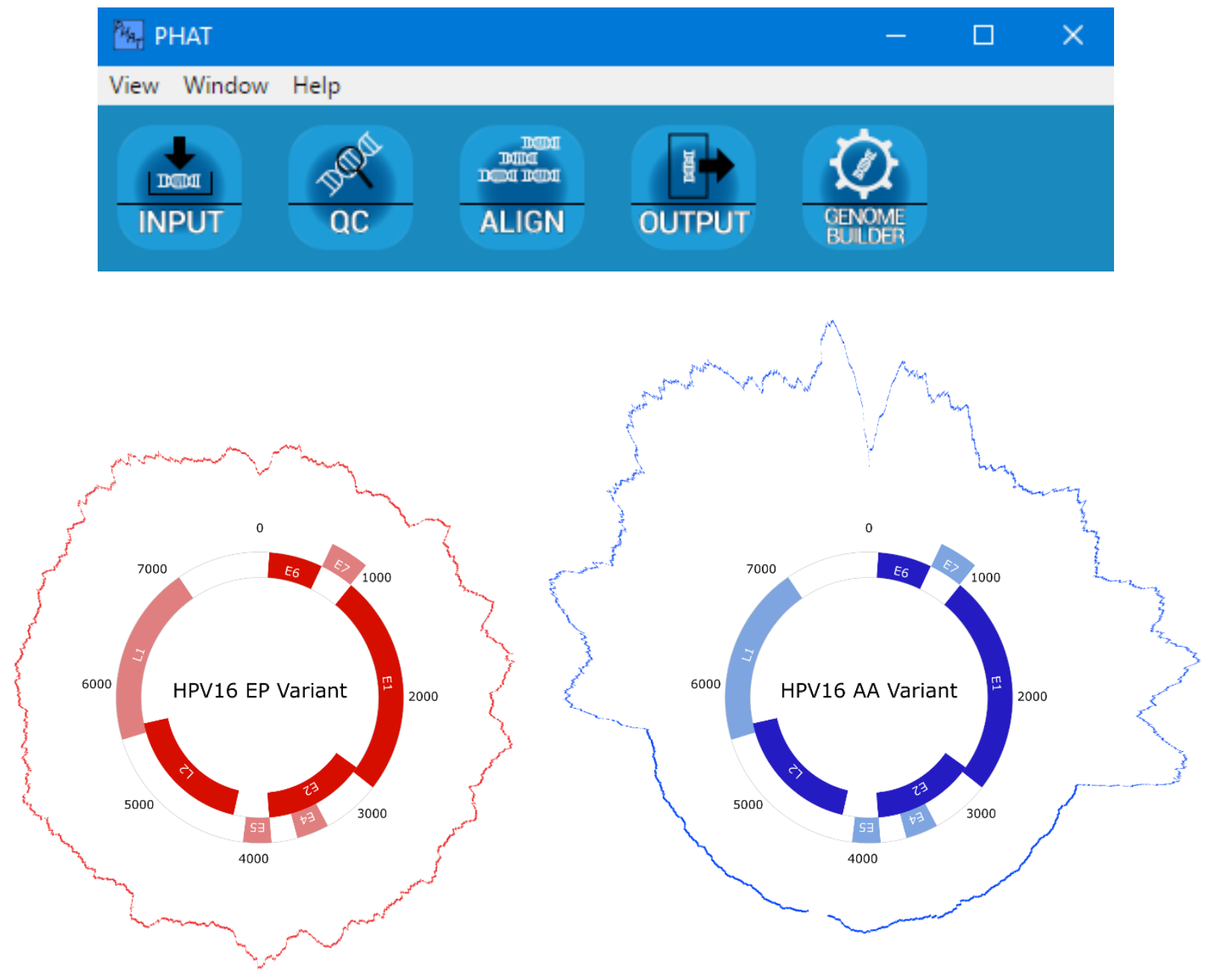

Figure 1. Pathogen-Host Analysis Tool (PHAT) tools and visualization. PHAT is equipped with tools (top image, showing the main toolbar) allowing the input of pathogen-containing nextgeneration sequencing data and reference sequences, quality control testing with FastQC, alignment with Bowtie2, SNP detection with VarScan 2, visualization with pileup.js and PHAT's genome builder, as well as tabular output. Example human papillomavirus type 16 (HPV16) genome maps and coverage plots were generated with PHAT to contrast coverage between viral variants: the European Prototype (EP) variant is episomal, whereas the Asian-American (AA) variant's coverage is disrupted by integration into host DNA. Data from Jackson et al., 2016. 


\section{Acknowledgements}

\section{Authors' Contributions}

The need for PHAT was initially conceived by Robert Jackson (RJ) and Ingeborg Zehbe (IZ). User interface (UI) and functional design was carried out by Christopher M. Gibb (CMG) and RJ collaboratively. Programming and technical planning was carried out by CMG. Manuscript and documentation writing and editing was carried out by CMG, RJ, Sabah Mohammed (SM), Jinan Fiaidhi (JF) and IZ. Intellectual property considerations made by SM and JF. Thanks to Zehbe lab members for user testing as well as students Mitchell Pynn, Jeremy Braun, Shane Liu, Zachary Moorman, and Nicholas Catanzaro for volunteering their time to technical improvements. We are thankful to the third-party developers of open-source tools that are incorporated in PHAT.

\section{Funding}

This work was supported by a Natural Sciences and Engineering Research Council of Canada (NSERC) grant to IZ (\#RGPIN-2015-03855) and NSERC Alexander Graham Bell Canada Graduate Scholarship-Doctoral (CGS-D) to RJ (\#454402-2014). The funding bodies had no role in study design, data collection, data analysis and interpretation, or preparation of the manuscript.

\section{References}

Afgan,E. et al. (2016) The Galaxy platform for accessible, reproducible and collaborative biomedical analyses: 2016 update. Nucleic Acids Res., 44, W3-W10.

Cock,P.J. et al. (2010) The Sanger FASTQ file format for sequences with quality scores, and the Solexa/Illumina FASTQ variants. Nucleic Acids Res., 38, 1767-1771.

Jackson,R. et al., (2016) Functional variants of human papillomavirus type 16 demonstrate host 
genome integration and transcriptional alterations corresponding to their unique cancer epidemiology. BMC Genomics, 17, 851.

Koboldt,D.C. et al. (2012) VarScan 2: somatic mutation and copy number alteration discovery in cancer by exome sequencing. Genome Res., 22, 568-576.

Langmead,B. and Salzberg,S.L. (2012) Fast gapped-read alignment with Bowtie 2. Nat. Methods, 9, 357-359.

Li,H. and Homer,N. (2010) A survey of sequence alignment algorithms for next-generation sequencing. Brief. Bioinform., 11, 473-483.

Li,H. et al. (2009) The sequence alignment/map format and SAMtools. Bioinformatics, 25, 20782079.

Li,Y. et al. (2014) ViRBase: a resource for virus-host ncRNA-associated interactions. Nucleic Acids Res., 43, D578-D582.

Lyons,E. and Freeling,M. (2008) How to usefully compare homologous plant genes and chromosomes as DNA sequences. Plant J., 53, 661-673.

Milne,I. et al. (2010) Tablet—next generation sequence assembly visualization. Bioinformatics, 26, 401-402.

Okonechnikov,K. et al. (2012) Unipro UGENE: a unified bioinformatics toolkit. Bioinformatics, 28, 1166-1167.

Vanderkam,D. et al. (2016) pileup.js: a JavaScript library for interactive and in-browser visualization of genomic data. Bioinformatics, 32, 2378-2379.

Xiang,Z. et al. (2007) PHIDIAS: a pathogen-host interaction data integration and analysis system.

Genome Biol., 8, R150. 